\title{
Estrategia pedagógica para el mejoramiento de los hábitos alimenticios
}

\author{
Pedagogical strategy to improve eating habits
}

Estratégia pedagógica para a melhoria dos hábitos alimentares

\author{
Míriam E. VelázquezTejeda \\ miriamv2002@yahoo.es \\ https://orcid.org/0000-0002-6245-6690
}

Universidad San Ignacio de Loyola, Lima-Perú

\section{Félix Fernando Goñi Cruz}

gonicruzf@gmail.com

https://orcid.org/0000-0001-5982-9858

Universidad San Ignacio de Loyola, Lima-Perú

\section{Marianela Luquillas Rodríguez \\ edithmaly@gmail.com \\ https://orcid.org/0000-1-2345-6789}

Universidad San Ignacio de Loyola, Lima-Perú

Artículo recibido 2 de septiembre 2021, arbitrado y aceptado 26 de octubre 2021 y publicado 30 de diciembre 2021

\section{RESUMEN}

El objetivo del estudio consiste en modelar una estrategia pedagógica que contribuya a la mejora de los hábitos alimenticios en los estudiantes de tercer grado de Educación Secundaria. La metodología empleada responde al paradigma socio crítico, el enfoque es cualitativo y se concreta en una investigación educacional de tipo aplicada. La muestra es intencionada y está constituida por un directivo, docentes, padres de familia, estudiantes y personal de servicio. Como parte del diagnóstico de campo se aplicaron diferentes técnicas como entrevistas, observación a clases y encuestas. Los resultados reafirmaron las deficiencias existentes en la práctica pedagógica evidenciadas en el bajo nivel de conocimientos, habilidades y hábitos alimenticios en los estudiantes. Como conclusión principal se aporta una estrategia pedagógica para contribuir a la promoción de los hábitos alimenticios saludables en los estudiantes de una institución educativa de secundaria de Perú.

Palabras clave: Estrategia pedagógica; Hábitos alimenticios; Nutrición; Promoción de hábitos alimenticios

ABSTRACT

\section{RESUMO}

The objective of the study is to model a pedagogical strategy that contributes to the improvement of eating habits in third grade students of Secondary Education. The methodology used responds to the socio-critical paradigm, the approach is qualitative and takes the form of an applied educational research. The sample is intentional and is constituted by a director, teachers, parents, students and service personnel. As part of the field diagnosis, different techniques such as interviews, classroom observation and surveys were applied. The results reaffirmed the existing deficiencies in the pedagogical practice evidenced in the low level of knowledge, skills and eating habits of the students. As a main conclusion, a pedagogical strategy is provided to contribute to the promotion of healthy eating habits in students of a secondary school in Peru.

Key words: Pedagogical strategy; Eating habits; Nutrition; Promotion of eating habits
O objetivo do estudo é modelar uma estratégia pedagógica que contribua para a melhoria dos hábitos alimentares dos alunos da terceira série do Ensino Médio. A metodologia utilizada responde ao paradigma sócio critico, a abordagem é qualitativa e toma a forma de uma pesquisa educacional aplicada. A amostra é proposital e é composta por um diretor, professores, pais, alunos e pessoal de serviço. Como parte do diagnóstico de campo, diferentes técnicas como entrevistas, observação em sala de aula e pesquisas foram aplicadas. Os resultados reafirmaram as deficiências existentes na prática pedagógica evidenciadas no baixo nível de conhecimentos, habilidades e hábitos alimentares dos estudantes. Como conclusão principal, uma estratégia pedagógica é fornecida para contribuir para a promoção de hábitos alimentares saudáveis em estudantes de uma escola secundária no Peru.

Palavras-chave: Estratégia pedagógica; Hábitos alimentares; Nutrição; Promoção dos hábitos alimentares 


\section{INTRODUCCIÓN}

El siglo XXI ha impulsado el desarrollo en las ciencias y el saber humano de manera que ha influido en los estilos de vida y en la alimentación de las personas. Los estudios reflejan la aparición de enfermedades crónicas como la obesidad, anorexia, bulimia y la desnutrición surgidas en su generalidad, por un desbalance en la ingesta de alimentos que afectan la salud nutricional y su calidad de vida en general.

La Organización Mundial de la Salud (OMS), se ha referido a la necesidad de implementar programas informativos y estrategias educativas que incidan en la conducta alimentaria de las personas. Ante ello, el Ministerio de Salud y el Ministerio de Educación de Perú, (2012), promulgaron la Resolución Ministerial 908-2008, donde se indica el expendio de alimentos sanos en las cafeterías escolares y al respecto decretó la Ley Nro.30021 que, promueve y argumenta la necesidad de ofrecerles alimentos saludables a los educandos.

El problema en los últimos años ha sido tratado por la OMS y distintos especialistas que, junto a los reportes médicos, abordan el problema de los hábitos alimentarios desde distintas aristas que influyen en la calidad de vida de las personas y afirman que, los estilos de vida saludables de los niños y los adolescentes son inadecuados para su salud y, por ende, para el desarrollo del aprendizaje. (Estrada, 2010 y De la cruz, 2012).

En ese sentido, el Diseño Curricular de Secundaria de Perú (2012) indica que la cultura alimenticia es un eje curricular transversal objeto de estudio de las disciplinas académicas, con el fin de contribuir a su formación en los estudiantes desde el contenido del sistema de clases. Sin embargo, la práctica pedagógica revela deficiencias en los docentes en el cumplimiento de ese objetivo, evidenciadas en la falta de métodos dialógicos y heurísticos que les permitan a los educandos reflexionar sobre el porqué es necesario ingerir alimentos sanos como vía para lograr la salud preventiva y desarrollar un aprendizaje de calidad.

El problema analizado se circunscribe en una institución educativa con estudiantes pertenecientes a la clase $\mathrm{B}$ y $\mathrm{C}$, que presentan dificultades en la alimentación por la falta de hábitos saludables. En la investigación participan no solo los educandos, sino también docentes, directivos, padres de familia y trabajadores de la cafetería institucional. El objetivo del estudio se orienta a la modelación de una estrategia pedagógica para contribuir a la mejora de los hábitos alimenticios en los estudiantes de tercer grado de Educación Secundaria de Perú.

El estudio es pertinente por cuanto trata el problema de la falta de hábitos alimenticios y cultura nutricional que incide en la calidad del aprendizaje y en la formación de los educandos para la vida. La problemática se trata desde la perspectiva sociocultural y didáctica y aporta como resultado del proceso investigativo una estrategia pedagógica que dados sus fundamentos teóricos incide en su tratamiento en la escuela y en la formación integral de los estudiantes.

\section{Reflexiones sobre la nutrición y la promoción de la salud}

Los estudios sobre la salud nutricional dan cuenta de su estado a nivel global y su incidencia en la calidad de vida de las personas. La nutrición involucra un conjunto de procesos fisiológicos del cuerpo humano que le permiten absorber, digerir, transformar, utilizar y asimilar los nutrientes contenidos en los alimentos. Es un proceso 
químico - biológico y fisiológico del organismo que es imposible controlarse como tal, lo que sí es controlable y por tanto educable, es la calidad del consumo nutricional para tener una vida sana y disposición por el estudio en el caso de los educandos (Rojas, 2011).

La nutrición es un proceso biológico de asimilación de los nutrientes alimenticios y la forma en que inciden estos en la salud y la calidad de vida de la persona. Los individuos sanos requieren distintos nutrientes durante el ciclo de vida a través de diferentes cantidades; sin embargo, los sujetos cubren las necesidades alimenticias a partir de los gustos individuales que han adquiridos por las influencias culturales. (Carro y Rebullido, 2016).

El proceso nutricional, la salud física, mentalemocional, sociocultural y espiritual se relacionan entre sí, incidiendo en la actitud, comportamiento y el desempeño de las personas. Al respecto Bruch, Malizia y Sorgentoni (2021) sostiene que, durante el crecimiento físico del niño y los adolescentes, el organismo aumenta y gasta mayor energía (Brown, 2008). Es un proceso que requiere de variedad en el consumo de proteínas, vitaminas y minerales porque las necesidades nutricionales son altas por el propio metabolismo en esas edades y el desgaste de energías en las actividades que realiza. (Miñana, Correcher y Dalmaus, 2016). De ahí que la escuela debe potenciar el conocimiento en los estudiantes para que sean conscientes del porqué se debe asumir una dieta variada, rica en nutrientes para crecer sanos y poder enfrentar las actividades con eficiencia (McKinney, 2013).

Al respecto, Carbajal (2013), propone un manual de nutrición en el que sugiere los alimentos que debe tener una dieta balanceada en post de una adecuada nutrición. Por ser un eje transversal del currículo debe tratarse en las clases, a fin de lograr gradualmente la cultura alimenticia en los estudiantes (Bargman, 2011; Díaz, Ficapal y Aguilar, 2015).

En ese sentido, el Ministerio de Salud de Perú (2019) en su programa de salud preventiva escolar, promueve la cantidad de alimentos a consumir por los estudiantes en los distintos momentos del día. Al respecto indica que un estudiante de edad escolar debe consumir la siguiente cantidad de calorías: $50 \%$ de proteínas de origen vegetal, 15\% proteínas de origen animal, $30 \%$ de grasas y $50 \%$ de hidratos de carbonos.

Congruente con ello, Carbajal (2013) y el Minedu (2019) refieren que los escolares en el día deben consumir cinco comidas: en el desayuno y merienda un $25 \%$, en el almuerzo un $30 \%$, merienda un $15 \%$ y en la cena un $25 \%$. Es recomendable que al término del día haya recibido el 55\% de calorías. Asimismo, la distribución de calorías debe ser de un 50\% de hidratos de carbono, un 30\% de grasas, con un $15 \%$ de proteínas de origen animal y $50 \%$ de origen vegetal.

En tal sentido, la escuela en particular está en el deber de concretar a través de los distintos procesos el fomento de la cultura en materia de salud preventiva y calidad de vida. Entendida la promoción de salud, según la carta de la Organización Panamericana de la Salud (1986), como el proceso que ofrece a la población los medios para ejercer un mayor control sobre su propia salud y cómo mejorarla. Es un proceso mediante el cual se capacitan las personas, se difunden los conocimientos, las conductas a seguir y los hábitos alimenticios sanos que deben ser practicados para desarrollar una salud de calidad. 
Por su parte, Bruch, Malizia y Sorgentoni (2021) precisan que, la promoción de salud conduce a potenciar la cultura alimenticia, el control de la salud, el autoconocimiento y en general, influye en la calidad de vida al igual que la práctica deportiva. Desde esta óptica se comprende la necesidad de establecer estrategias mediadoras entre los organismos encargados, la población y su entorno, a fin de incorporar la responsabilidad social de la salud a la actuación individual en la práctica.

\section{Los hábitos alimenticios y su incidencia en la enseñanza-aprendizaje}

El proceso de enseñanza- aprendizaje es un proceso organizado, planificado, dirigido, cultural y comunicativo donde confluyen leyes, principio y teorías de forma interdisciplinaria, en función de lograr la unidad dialéctica entre la instrucción, la educación y el desarrollo integral de los estudiantes en sus formas de pensar, hacer y sentir. (Rico, Santos y Martín, 2013 y Addine, et al. 2015).

Un principio pedagógico de la clase es la atención preventiva a la salud, que por su carácter transversal devienen en una herramienta gnoseológica, valorativa y práctica que se orienta a la formación de la cultural nutricional y los hábitos saludables como garantía para la salud escolar y el aprendizaje exitoso de los educandos. En ese sentido se impone potenciar el conocimiento del porqué se deben formar los hábitos de vida saludable como se precisa en los enfoques teóricos, publicaciones, principios, conferencias y discusiones científicas a nivel mundial. (Muñoz, Fernández y Navarro, 2015; Ojeda, Muñoz y Menéndez, 2019).

Los hábitos alimentarios abordados desde el punto de vista sociocultural, se asumen como la adquisición de conductas conscientes en los distintos contextos de actuación. Es la forma en que el sujeto asimila su entorno y elige los alimentos para su consumo haciendo uso del conocer, hacer, actuar y ser unido al aprendizaje asumido en los diferentes periodos de la vida (Vygotsky, 1978).

Acerca de los hábitos alimentarios Laguna (2015), asevera que, son manifestaciones recurrentes conscientes del comportamiento individual y colectivo respecto al qué, cuándo, dónde, cómo, con qué y con quién consumir alimentos hasta interiorizarlo y adoptarlos de manera directa e indirectamente como parte de sus prácticas socioculturales.

En ese orden, Torres, Solís, y Rodríguez y Mogel (2017), refieren que, los hábitos alimentarios son expresiones auténticas de elección de los nutrientes que desea consumir el sujeto. Estos se aprenden desde las primeras edades y las experiencias que se adquieran a partir de los conocimientos, costumbre, ejemplo de los padres y la labor de la escuela, que se van fomentando en la medida que el sujeto enriquece su cultura alimenticia y general durante la vida.

Sobre los hábitos alimentarios, las conductas alimentarias y los estilos de vida poco saludables de los adolescentes se han referido distintos estudios que permite analizar, reflexionar y exhorta a revertir la realidad a fin de favorecer la calidad de vida de las personas (Greppi, 2012).

Se reconoce en la práctica que la tradición culinaria familiar y la cultura de los contextos son esenciales para iniciar y mantener los hábitos alimenticios en los estudiantes de una sociedad. Es a través de la socialización y las influencias socioculturales recibidas donde se aprende a consumir y se adquieren nuevas preferencias en la medida que se amplía el campo de conocimiento, las 
experiencias, la interacción y la cultura en general. (Acosta, Buitrago y Parra, 2018).

La escuela tiene el encargo social de contribuir a la formación integral del educando; ello precisa del docente maestría al tratar el contenido desde un enfoque interdisciplinario y transdisciplinario para generar la problematización, asumir posiciones y producir nuevos significados (Addine, 2015). En la clase se pueden presentar situaciones pedagógicas sobre la cultura alimenticia, con la intención de generar el debate acerca de la misma, su influencia en la salud preventiva y en la calidad del aprendizaje, con el objetivo de concientizar qué actitud asumir y por qué. Para lograrlo deberán aplicarse las metodologías activas que estimulan la crítica, la autorreflexión, la autorregulación y el aprendizaje metacognitivo en los estudiantes. (De Corte, 2015; Navarro, 2015; Moreno y Velázquez, 2017).

En resumen, los hábitos alimentarios son procesos aprendido por el sujeto en la interacción social al relacionarse con los otros como parte de la cultura contextual y la escuela en particular. Primero en el hogar y luego en la institución educativa se va tomando conciencia y adquiriendo conocimientos en la medida que se sistematiza como parte del contenido de las clases. Ello orienta al educando a formar y desarrollar una actitud consecuente a través de las actividades de aprendizaje en las que va alcanzando niveles progresivos de desarrollo en la medida que amplía su cultura escolar y alimenticia en particular. (Navarro, Vera, Munguía, Ávila, Sánchez y Hernández, 2017).

La cultura alimentaria, entendida como el proceso de adquisición de los conocimientos, tradiciones, costumbres, interacción social y elaboraciones simbólicas de los alimentos por las personas; es un proceso donde influye el ciclo productivo de los alimentos de un contexto determinado (Calderón, Taboada, Argumedo, Ortiz, López y Hernández, 2017).

Es un imperativo aplicar actividades variadas en la enseñanza- aprendizaje y en los diferentes procesos formativos que orienta la escuela donde se argumente por qué se debe consumir alimentos sanos, y practicar deportes como premisa para garantizar la salud preventiva y la eficiencia educativa. El tratamiento didáctico, dialógico y persuasivo conducirá a la comprensión e incidirá en el desarrollo intelectual, físico, social y motivacional de los estudiantes. Se trata de ejercitar las habilidades y formar hábitos en función de lograr actitudes conscientes, autorreflexivas y participativas. Para ello el docente creará situaciones pedagógicas donde se problematice para saber cómo piensan los estudiantes y asuman posiciones adecuadas.

\section{MÉTODO}

La metodología empleada respondió al paradigma socio crítico, el enfoque es cualitativo y responde a una investigación educacional de tipo aplicada. En ella se integran los métodos cuantitativos y cualitativos en la búsqueda de la realidad para analizar las causas que inciden en el objeto de estudio y proponer el cambio. El problema investigado se identificó en el desempeño docente del aula, se sistematizan los fundamentos teóricos, contrastados con los resultados del diagnóstico de campo y se identificaron las categorías emergentes incidentes en el problema. A partir de esas aproximaciones sucesivas, se propuso como producto de la investigación una estrategia pedagógica para contribuir al desarrollo de los hábitos alimenticos de los estudiantes. 
El diagnóstico de campo se aplicó en una institución educativa de Secundaria mediante una muestra seleccionada de forma intencionada, constituida por 15 estudiantes del cuarto grado, un directivo pedagógico, 10 docentes de la disciplina, Educación para el trabajo y la Salud, 15 padres de familia, y dos trabajadores de la cafetería institucional.

Se emplearon distintos instrumentos un cuestionario a los estudiantes con el propósito de conocer el nivel de conocimientos sobre la alimentación; una guía de entrevista semiestructurada a los docentes y directivos a fin de constatar los conocimientos y las habilidades profesionales sobre el problema; guía de observación a clases para constatar el tratamiento del objetivo en el proceso de enseñanza y aprendizaje; guía de entrevista a los padres de familia y a los trabajadores de la cafetería. La estrategia propuesta como producto científico, fue validado por un equipo de expertos en la materia.

\section{RESULTADOS Y DISCUSIÓN}

Primer momento: Comenzó con el proceso de transcripción y codificación de los datos obtenidos; se describieron las acciones y los comentarios obtenidos en la entrevista y la observación a clases a docentes; el cuestionario aplicado al directivo, y a los trabajadores de la cafetería, la encuesta; la prueba pedagógica aplicada a los estudiantes y la entrevista a los padres de familia. Se ordenó la información registrada, agrupándola por instrumento y se efectuó la transcripción de los datos. Luego se trabajó en las tablas la codificación concerniente a la identificación de ideas clave (códigos), y los datos cuantitativos se analizaron en las tablas de frecuencia, que permitieron determinar las tendencias y regularidades.

En esta parte se analizó el resultado de la prueba pedagógica y la encuesta aplicada a los15 estudiantes, cuyos resultados revelan el estado de los indicadores: cultura alimentaria, hábitos alimentarios y salud nutricional.

En el indicado cultura alimentaria: nueve estudiantes (69\%), demostraron un bajo nivel de los conocimientos sobre las tradiciones alimenticias, las costumbres, los alimentos sanos y los tipos de alimenticios que ayudan a la salud preventiva; cuatro estudiantes (26.67\%), alcanzan un nivel medio, dominan algunos aspectos de las tradiciones alimenticias, costumbres sobre los alimentos sanos que le explica su familia y los tipos de alimenticios que ayudan a la salud preventiva; y dos de ellos (13.33\%) alcanzan un alto nivel en los conocimientos sobre las tradiciones alimenticias, costumbres, los alimentos sanos y los tipos de alimentos ayudan a la salud preventiva.

En cuanto al indicador hábitos alimentarios: 10 estudiantes ( 66.67\%) manifestaron un bajo nivel de los hábitos alimenticios, consumo de gaseosas, sándwich, dulces, pizzas y alimentos chatarras, argumentando que les encuentran más sabor que la comida de la casa; no conocen los nutrientes de los alimento y no tienen hábitos alimentarios sanos; cuatro estudiantes (26.67\%), reflejaron un nivel medio en los hábitos alimenticios, consumen gaseosas, sándwich, dulces, pizzas, alimentos chatarras, argumentando que, a veces tienen más sabor que algunas comida de la casa; conocen los nutrientes de algunos alimentos y como positivo, se interesan por desarrollar hábitos alimenticios sanos; y uno de ellos (13.33\%), reflejaron un nivel alto, 
tienen hábitos alimenticios adecuados, respetan los horarios de comida, consumir bastante agua, verduras, frutas y otros nutrientes sanos y explicaron que sus padres les han enseñado los beneficios de la alimentación sana y nutritiva para la vida.

En el indicador salud nutricional: nueve estudiantes (60\%), evidenciaron un bajo nivel de conocimientos, acerca del valor de una alimentación nutritiva, hábitos alimenticios positivos, una dieta equilibrada combinada con la práctica de ejercicio físico como garantía para tener una salud; cuatro estudiantes (26.67\%), tienen un nivel medio, al referir que es importante la alimentación nutritiva, hábitos alimenticios positivos, aunque no reconoce que la dieta debe basarse en verduras, frutos, avenas, carnes y otros alimentos portadores de altos nutrientes ni la práctica de ejercicios para lograr una adecuada salud; y dos estudiantes (13.33\%), demostraron un nivel alto de conocimientos sobre la importancia de la alimentación nutritiva, hábitos alimenticios positivos como es una dieta basada en verduras, frutos, avenas, carnes y otros alimentos portadores de nutrientes y realizar la práctica de ejercicios diario para lograr una adecuada salud (Figura 1).

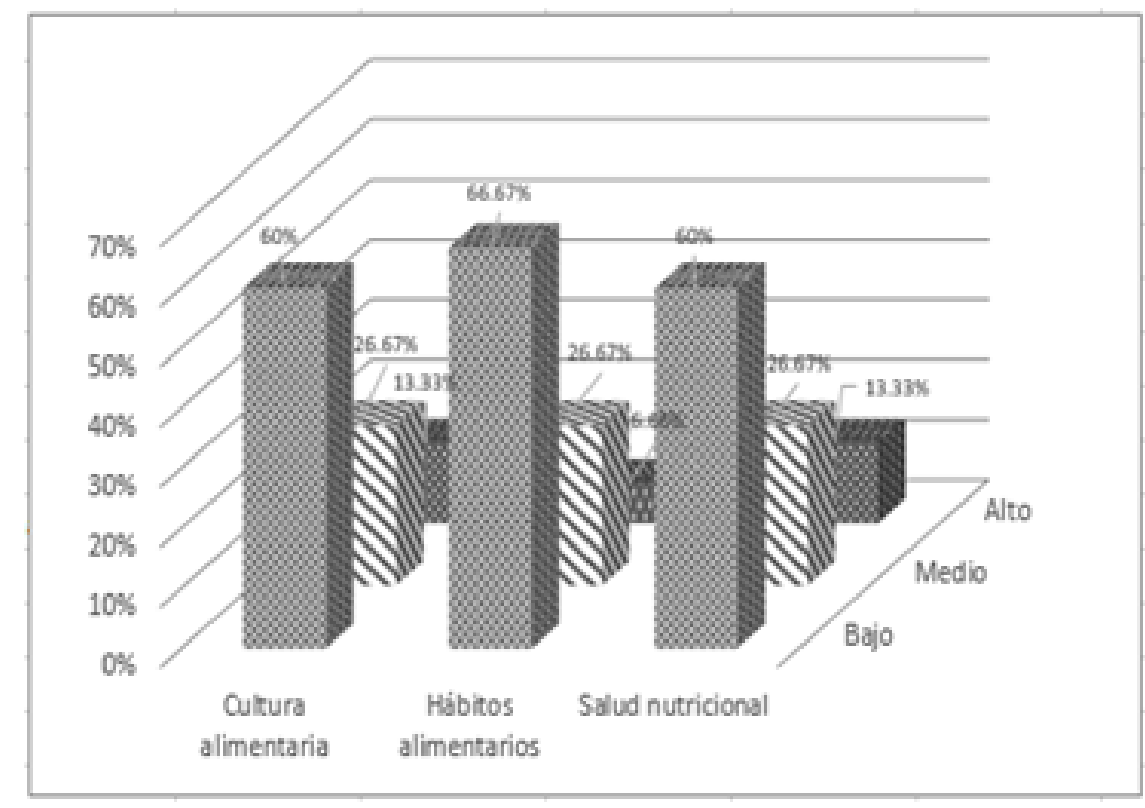

Figura 1. Resumen del estado de los indicadores en los estudiantes.

Segundo momento: Se compararon y clasificaron las categorías mediante la integración de la información acopiada y se arribó a las conclusiones aproximativas a partir de la categoría apriorística: conocimientos de la alimentación saludable y las subcategorías: cultural alimentaria, hábitos alimentarios y salud nutricional, como se lustra (Figura 2). 


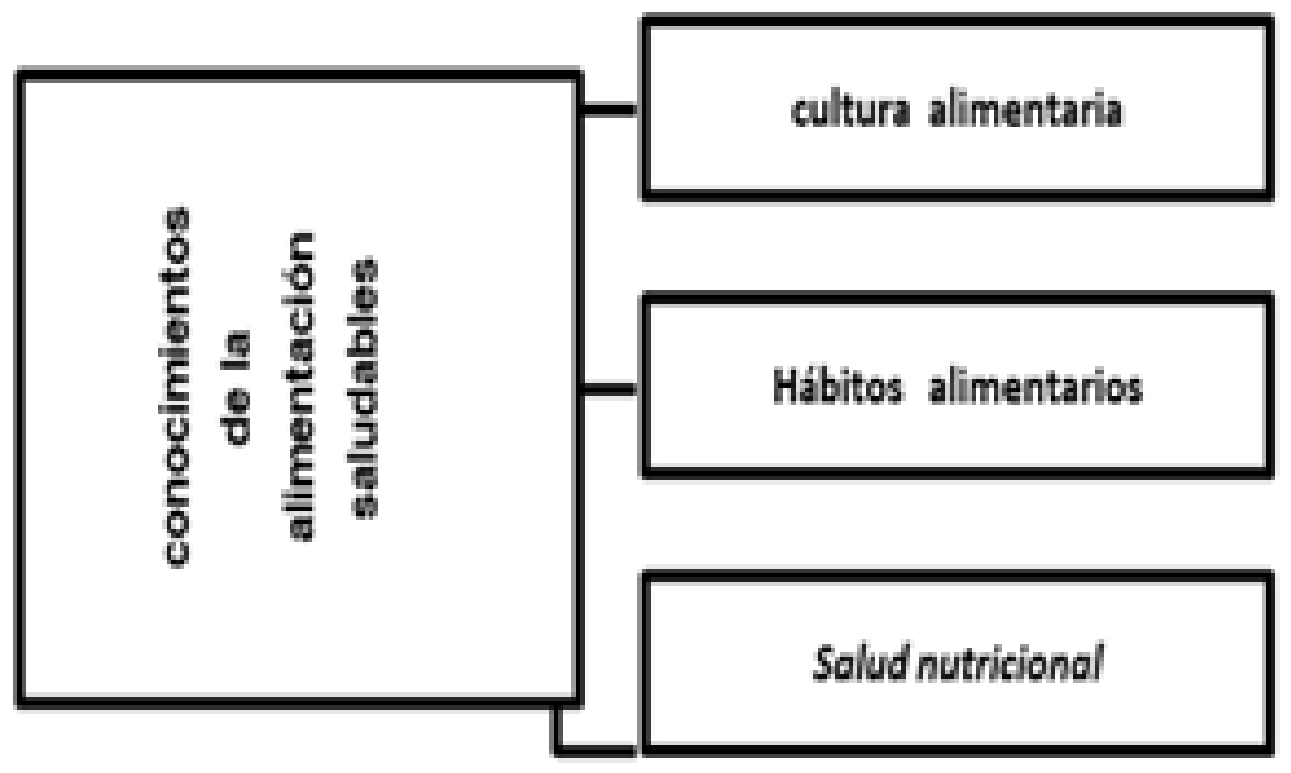

Figura 2. Categoría y subcategorías apriorísticas.

Tercer momento. Se procedió a la triangulación, discusión e interpretación de los resultados obtenidos.

Al triangular la información se constató que, el 95\% de los docentes en las entrevistas consideran importante tratarla cultura alimentaria desde el contenido de las clases como un eje metodológico transversal, lo cual revela una contradicción entre el discurso y la práctica pedagógica, pues las clases observadas evidenciaron deficiencias en los conocimientos sobre la salud preventiva y falta de aplicación de métodos que conlleven a problematizar y reflexionar sobre los hábitos alimentarios y la salud preventiva.

En las entrevistas, los directivos, los trabajadores de la cafetería y las familias, no reconocen desde su frente respectivo, que la salud alimentaria y la promoción de salud responde al programa de prevención de salud escolar establecido a nivel nacional y que, debe incluirse en el quehacer de los procesos docente -educativo que lleva a cabo la escuela, a fin de contribuir a la formación y desarrollo de la cultura alimenticia en los estudiantes.

Los estudiantes, revelaron un bajo nivel de conocimientos y actitudes sobre la cultura alimentaria, evidenciado en la falta de información de alimentos sanos, en el consumo de alimentos chatarra, exceso de refrigerios, incorrectos horarios de alimentación que sustituyen por sándwich, dulces, pizzas y refrescos, afectándose así su salud nutricional y su rendimiento escolar.

Al concluir el análisis se analizaron las contradicciones, las similitudes y las categorías emergentes causantes del problema investigado, cuyos resultados se refleja a continuación (Figura $3)$. 


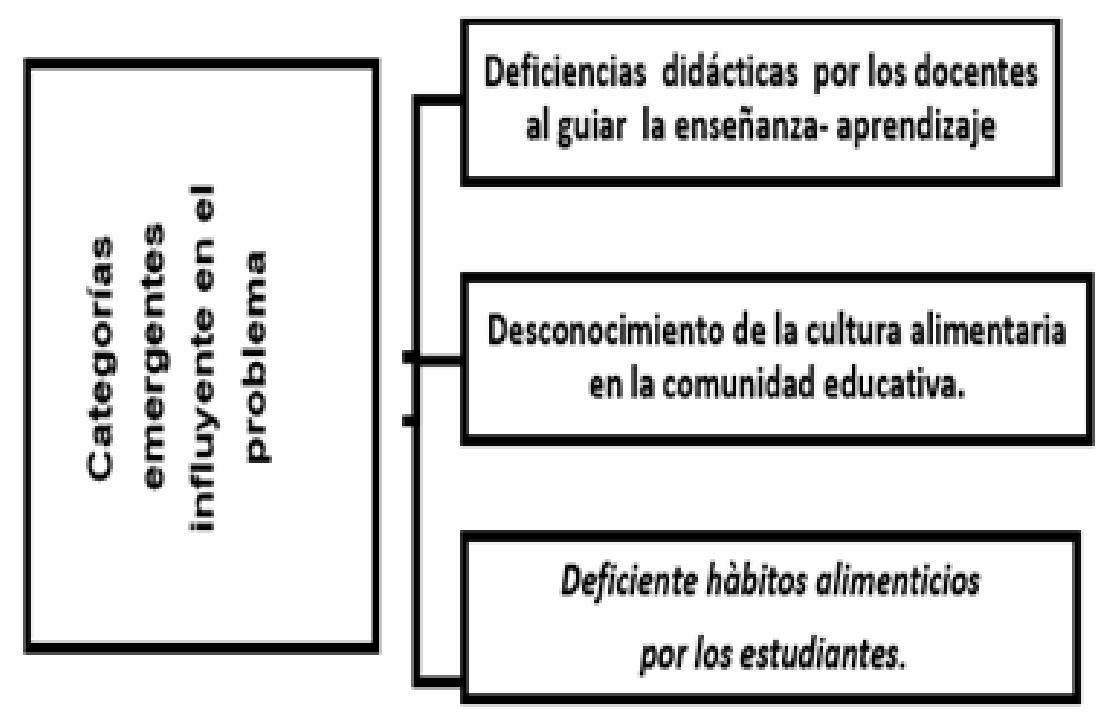

Figura 3. Categorías emergentes causantes del problema investigado.

$\mathrm{Al}$ identificarse las categorías emergentes influyentes en el problema investigado, se procedió a modelar la estrategia pedagógica a fin de contribuir a la transformación.

Modelo de estrategia pedagógica para el mejoramiento de los hábitos alimentarios

El colectivo pedagógico de la institución educativa tiene como responsabilidad, poner en práctica un sistema de actividades creativas y métodos activos que incidan en el pensamiento, la formación y desarrollo de los conocimientos sobre la cultura nutricional con el propósito de potenciar la ejercitación de los hábitos de alimentación sana como vía para lograr la salud preventiva y un rendimiento eficiente en los estudiantes. (Addine, 2015).

La estrategia pedagógica diseñada tiene como propósito la capacitación teórica y metodológica de los directivos, los docentes, los trabajadores de servicio y los padres de familia con el fin que ahondar en los conocimientos sobre la cultura alimentaria, los hábitos alimentarios y la promoción de la salud y en consecuencia que los estudiantes alcancen los niveles de desarrollo requeridos a través de todos los procesos que se realizan en la escuela.

\section{Procesos que integra la estrategia propuesta}

La estrategia pedagógica se ha modeló a partir de un conjunto de actividades como: reuniones metodológicas, charlas, debates, talleres, acompañamiento pedagógico, presentación de clases modelo, concursos y exposiciones que serán realizadas por un especialista, cuyo objetivo principal es la capacitación de los docentes, los padres de familias, los trabajadores de servicio y los directivos orientadas a profundizar en la cultura alimenticia, los hábitos y la salud nutricional para potenciar la salud preventiva en los estudiantes.

La estrategia propuesta está estructurada a través de los siguientes procesos:

Proceso de concertación. Se realiza la capacitación a fin de lograr su participación consciente en la aplicación del programa. Sus pasos son: capacitación de la comunidad educativa a través de talleres, 
divulgación de volantes, emisiones del programa de la Radio Base y la firma de compromisos de los miembros de la comunidad educativa.

Proceso de diagnóstico y planificación. Se realiza el diagnóstico y caracterización del estado actual; planificación de las acciones orientadas a la transformación. Sus pasos son: de la situación real, elaboración del plan, asignar un presupuesto para cubrir los gastos materiales y conformar un equipo especializado en los temas de salud nutricional.

Proceso de intervención. Aplicación de las acciones teóricas y metodológicas a través de un cronograma de actividades para fortalecer la promoción de los hábitos alimentarios saludables y difundir los resultados.

\section{Orientaciones dirigidas a los docentes}

Se sugiere poner en práctica el acompañamiento pedagógico, como una forma de superación que garantiza la actualización profesional desde el puesto de trabajo a partir de las potencialidades de otros docentes más preparados de la institución. Esta modalidad le permitirá alcanzar el nivel de profesionalismo en la formación de la cultura alimenticia de los educandos, desde la propia clase (Zegarra y Velázquez, 2016).
Para ello se debe diseñar un plan de capacitación con las actividades y el contenido de la salud alimenticia y cultura nutricional que tratará en las clases, los métodos a emplear, las actividades de aprendizaje a realizar, los materiales a usar, cómo organizar las clases y el rol de guía, facilitador y orientador que el asesor, trabajará con los docentes mediante el enfoque interdisciplinario de la clase.

En la asignatura Educación para el Trabajo y la Salud, se propone orientar la enseñanza y aprendizaje desde el enfoque Sociocultural de Vygotsky (1978), que integrado a los fundamentos de la cultura alimentaria de (Laguna, 2015), estimulan la observación, la indagación, el procesamiento de la información, la reflexión persuasiva y la aplicación del conocimiento en las actividades práctica. Esta forma de enseñanza incide en el pensamiento crítico, en el autoaprendizaje y la autorregulación del estudiante (Navarro, 2015).

Proceso de monitoreo y evaluación. El control para constatar la eficiencia del programa; verificar los logros y las dificultades presentada a fin de reajustar las acciones hasta alcanzar los objetivos propuestos. Su aplicación requiere de los siguientes pasos: Creación de la comisión de evaluación, elaboración y aplicación de la matriz de evaluación y la redacción del informe final. (Figura 4 y Tabla 1). 


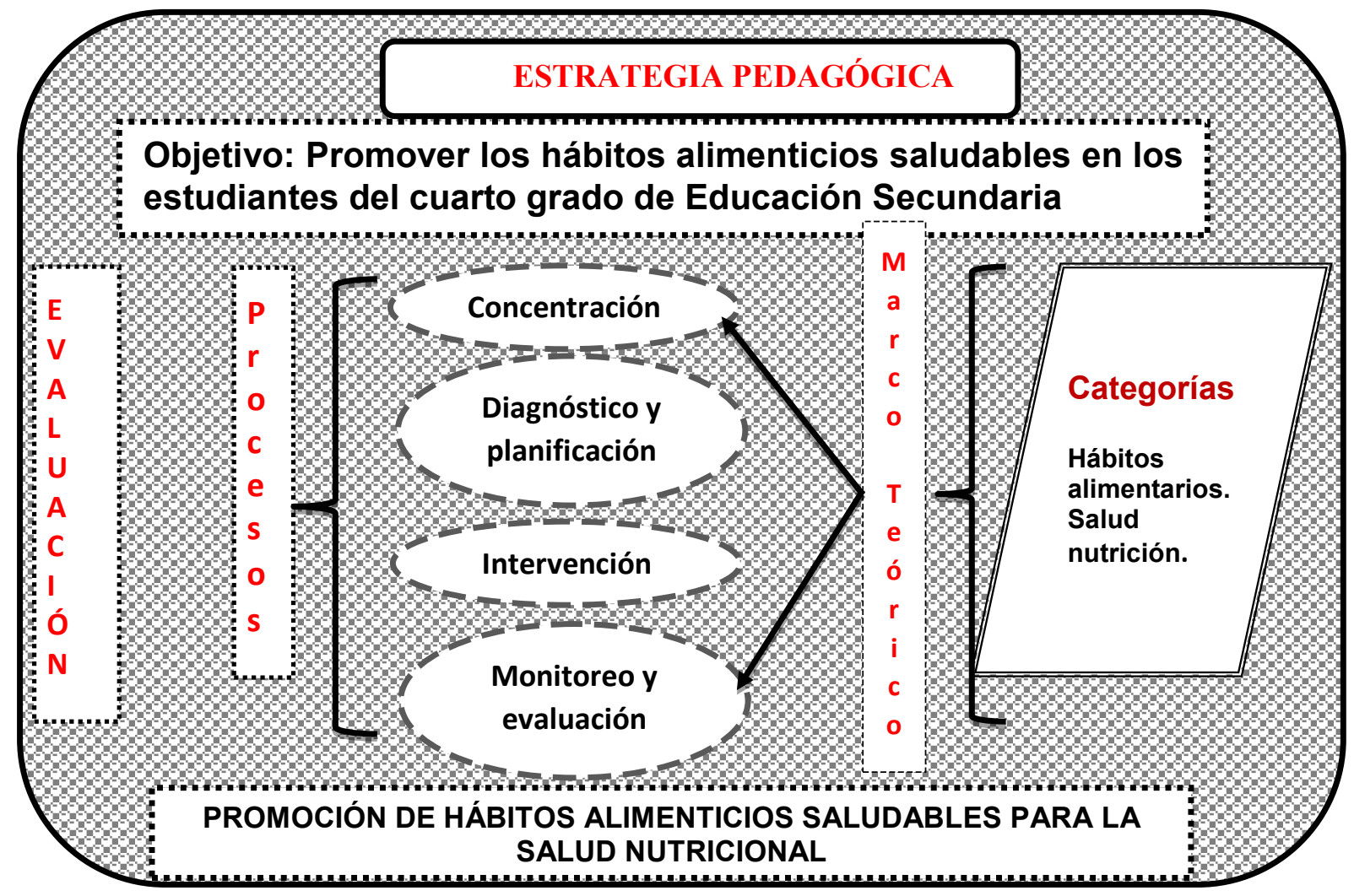

Figura 4. Crocesos de la estrategia modelada.

Tabla 1. Estructura de la estrategia pedagógica propuesta.

\begin{tabular}{|c|c|c|c|c|}
\hline Competencia & Capacidad & Indicadores & Actividades & $\begin{array}{c}\text { Comunidad } \\
\text { Educativa }\end{array}$ \\
\hline
\end{tabular}

Utiliza

conocimientos

científicos

para explicar

los hechos,

fenómenos

naturales

, tomar

decisiones

y plantear

alternativas de

solución.
Toma decisiones

y plantea

alternativas de

solución con

argumentos

científicos para

cuidar la salud.
Elige entre varias opciones, con el fin de hacer frente a situaciones de la vida cotidiana, en diferentes contextos y a nivel personal, familiar, social y otros.
Talleres de superación personal y gestión. Directivos

Guía de estrategias.

Docentes

Escuela de Padre.

Talleres de capacitación.

Guía metodológica

Modelos de sesión de clase.

Personal de la cafetería

Charla y conversatorios.

Divulgación en los murales Estudiantes. 
Validación de la estrategia por criterios de especialistas

Para evaluar la estrategia diseñada se empleó el método de juicio de expertos donde se emplearon dos rúbricas de valoración: aspectos externo e interno del producto científico. Este método tiene como requerimiento para su aplicación, que los especialistas ostenten determinados por los siguientes requisitos: poseer el grado de Maestro o Doctor en Educación y ser especialistas de Nutrición.

Durante el proceso de la validación cuantitativa, se evaluaron diez criterios para señalar el valor que le asignaban al producto a partir del siguiente puntaje: 1 deficiente, 2 baja, 3 regular, 4 bien y 5 muy bien, como se muestra en la Tabla 2 y 3.

Tabla 2. Escala de valoración.

\begin{tabular}{lc}
\hline Escala & Valoración \\
\hline $0-25$ & Deficiente \\
$26-59$ & Baja \\
$60-70$ & Regular \\
$71-90$ & Bien \\
$91-100$ & Muy Bien \\
\hline
\end{tabular}

Fuente: Staff de investigadores de la USIL (2016).

Tabla 3. Sumatoria de valoración total

\begin{tabular}{ccc}
\hline Sumatoria de valoración total & Promedio de valoración & Valoración literal \\
\hline 284 & 95 & Muy Bien \\
\hline
\end{tabular}

Fuente: Staff de investigadores de la USIL (2016).

Los expertos concluyeron otorgando la calificación de muy bien al producto aportado por la investigación; enfatizaron en el rigor científico de la propuesta y que su basamento pedagógico permite su aplicabilidad en la institución del nivel de Secundaria y añadieron que, según los sustentos teóricos, actualidad y necesidad del tema puede aplicarse en otras áreas curriculares de secundaria e instituciones.

\section{CONCLUSIONES}

Las investigaciones analizadas y la sistematización teórica realizadas confirman que el consumo de alimentos saludables propicia la salud nutricional que influye en la masa corporal y el equilibrio nutricional necesario, para activar los neurotransmisores encargados de estimular las capacidades y el aprendizaje en los estudiantes, y revelan que el sobrepeso y la obesidad en la edad 
infanto-juvenil, es un problema actual de la salud pública, la sociedad y de la educación en particular.

Los directivos entrevistados consideran que los estudiantes no han desarrollado los hábitos alimenticios porque la familia practica una dieta nutricional que influye negativamente en los hijos; apreciándose así que no identifican la responsabilidad de la escuela en la promoción de la salud alimenticia como un eje curricular metodológico que la institución debe sistematizar para potenciar el tratamiento a la cultura alimenticia como parte del contenidos de la enseñanza desde las áreas curriculares del plan de estudio a fin de contribuir a la formación de los educandos.

El diagnóstico evidenció en los estudiantes un bajo nivel en los conocimientos, las habilidades y los hábitos alimentarios, evidenciados en el consumo de alimentarios de bajo nivel nutricional que inciden negativamente en la salud preventiva, rendimiento académico y en sus actitudes de forma general.

Los docentes demuestran desconocimiento teórico del tema objeto de estudio y, por ende, no aplican los métodos y las estrategias metodológicas en las clases que les permita problematizar con los estudiantes con la intención de que estos analicen y reflexionen desde el objeto de estudio de la enseñanza a fin de que opinen, valoren, debatan, critiquen, asuman posiciones responsables y se orienten permanentemente en la formación de los hábitos alimentarios saludables.

El aporte esencial de la investigación es de carácter teórico-práctico y se concreta en una estrategia pedagógica, cuyo propósito es involucrar y potenciar la preparación de la comunidad educativa en la cultura alimenticia, estimular los hábitos alimentarios y la salud nutricional a través de todos los procesos formativos que realiza la institución educativa.

La estrategia pedagógica propuesta, se ha fortalecido en los fundamentos teóricos y prácticos de las ciencias pedagógicas y por tal razón puede ser aplicada no solo en la institución de secundaria, sino en otras afines como una alternativa para contribuir a la capacitación de la cultura alimenticia en la comunidad pedagógica con el objetivo de favorecer la formación de los hábitos alimenticios en los estudiantes.

Validar el impacto de la puesta en práctica de la estrategia modelada, comprobar su contribución en la cultura alimenticia, la formación de hábitos, la salud nutricional y el aprendizaje de los estudiantes a fin de realizar los reajustes correspondientes.

Presentar los resultados del presente estudio en los distintos eventos científicos programados a fin de socializarlos y continuar la investigación en otras aristas del problema de la salud preventiva y su incidencia en el aprendizaje para aportar a la práctica profesional.

REFERENCIAS

Acosta, L; Buitrago, A. y Parra, C. (2018). Hábitos alimenticios en niños y niñas escolares en una institución educativa privada del norte de Bogotá. Revista pediatría, 51(1), DOI: https:// doi.org/10.14295/pediatr.v51i1.110

Addine, F., Álvarez, L., Martínez, M., Parra, I., Sierra, R. y Gutiérrez, M. (2015). Didáctica de la pedagogía y la Psicología. La Habana: Pueblo y Educación

Bargman, D. (2011). Educación alimentaria y nutricional, libro para el docente. Serie Ciencia, Salud y Ciudadanía. Proyecto de Alfabetización Científica, Argentina 
Brown, J. (2008). Nutrición en las diferentes etapas de la vida. México: Edamsa Impresiones S.A de C.V

Bruch, V., Malizia, R. y Sorgentoni, S. (2021). Educación alimentaria nutricional (EAN) como contenido transversal en perspectiva de política pública en instituciones de nivel inicial y primario de la ciudad de Rosario, Provincia de Santa Fe, Revista de la Escuela de Ciencias de la Educación, 1(16), 107-117, https:// revistacseducacion.unr.edu.ar/index.php/ educacion/article/view/591/411

Calderón, M, Taboada, R, Ortiz, E, López, A, y Hernández, J. (2017). Cultura alimentaria: Clave para el diseño de estrategias de mejoramiento nutricional de poblaciones rurales. Agricultura, sociedad y desarrollo, 14(2), 303-320. http://www.scielo.org.mx/ scielo.php?script $=$ sci_arttext\&pid $=\mathrm{S} 1870$ 54722017000200303\&lng=es

Carbajal, A. (2013). Manual de nutrición y dietética. Departamento de nutrición. Facultad de Farmacia. Universidad Complutense de Madrid. https://www.ucm.es/nutricioncarbajal/

Carro, L., y Rial Rebullido, T. (2016). Hábitos alimentarios y de actividad física de alumnado de Educación Primaria: Estudio descriptivo de un colegio de Pontevedra. Sportis, 2(1), 77-92

De la Cruz, E. (2012). Cristal de agua para el abordaje de educación alimentaria y nutricional en educación inicial. Revista de Investigación, $36(77)$

De Corte, E. (2015). Aprendizaje constructivo, autorregulado, situado y colaborativo: un acercamiento a la adquisición de la competencia adaptativa (matemática). Revista Páginas de Educación, 8(2). http://revistas.ucu.edu. uy/index.php/paginasdeeducacion/article/ view/690/

Díaz, T.; Ficapal, P. y Aguilar, A. (2015). Hábitos de desayuno en estudiantes de primaria y secundaria: posibilidades para la educación nutricional en la escuela. Nutrición Hospitalaria, 33(4), 909-914. doi: http://dx.doi.org/10.20960/ nh.391
Estrada, D. (2010). Hábitos alimentarios y factores culturales en mujeres embarazadas que acuden a consulta externa del hospital básico Dr. Montenegro del Cantón. Chillanes, Bolívar, 2010. (Tesis de grado). Riobamba, Ecuador: Escuela Superior Politécnica de Chimborazo. http:// dspace.espoch.edu.ec/handle/123456789/1062.

Greppi, D (2012). Hábitos alimentarios en escolares adolescentes. Tesis para optar la licenciatura en Nutrición. Universidad Abierta Interamericana. http://imgbiblio.vaneduc.edu.ar/fulltext/files/ TC111865.pdf

Laguna, A. (2015). Hábitos alimenticios, peso y metabolismo. UAEMéx, México

Ministerio de Salud de Perú. (2012). Guía técnica de gestión de promoción de la salud en instituciones educativas para el desarrollo sostenible. http:// www.fhspereclaver.org/userfiles/file/guia nutricion_saludable.pdf.

Ministerio de Salud. (2019). Resolución Ministerial 908- Minsa, Lima. http://www.munizlaw.com/ normas/2012/noviembre/17-11-12/r.m.\%20 n\%c2\%ba\%20908-2012-minsa.pdf

Ministerio de Educación de Perú (2012). Diseño Curricular Nacional de Secundaria Básica

McKinney, C. (2013). Assessment of Dietary Behaviors of College Students Participating in the Health Promotion Program BUCS: Live Well. Electronic Theses Dissertations. ht tp://dc.etsu.edu/cgi/viewcontent. cgi? article $=2265 \&$ context $=$ etd

Miñana, Correcher y Dalmaus (2016). La nutrición del adolescente. ADOLESCERE. Revista de Formación Continuada de la Sociedad Española de Medicina de la Adolescencia, 4(3), 6-18

Moreno, W. y Velázquez, M. (2017). Estrategia didáctica para desarrollar el pensamiento crítico. Revista Iberoamericana sobre Calidad, Eficacia y Cambio en Educación Reice, 15(2), 53-73. https://doi.org/10.15366/reice2017.15.2.00315.

Muñoz, A., Fernández, N., Navarro, R. (2015). Estudio descriptivo sobre los hábitos saludables en alumnado de Primaria desde la Educación Física escolar. Universidad de Santiago de 
Compostela. SportisScientificTechnicalJournal, 1 (1), 87-104

Navarro, M. (2015). El efecto del aprendizaje activo en el desarrollo de habilidades propias del pensamiento crítico en estudiantes de ciencias. Revista de Psicodidáctica, 20(2), 209-226.http:// www.ehu.eus/revista-psicodidactica

Navarro, A., Vera, O., Munguía, P., Ávila R., Sánchez, M., Ochoa, C., y Hernández, P. (2017). Hábitos alimentarios en una población de jóvenes universitarios (18-25 años) de la ciudad de Puebla. Revista Española de Nutrición Comunitaria, 23(2), 31-37.doi: 10.14642/ RENC.2017.23.sup2.5176

Organización Panamericana de la Salud (1986). Carta de Ottawa para la Promoción de la Salud. Ottawa, Canadá

Ojeda, M., Muñoz, J. y Menéndez, P. (2019). Hábitos de vida saludables y rendimiento escolar en estudiantes universitarios. Revista de la Escuela de Ciencias de la Educación, 15(2), 34-44. ISSN 2362-3349. https://doi.org/10.35305/rece. v2i15.544

Rico, P., Santos, E. y Viaña, V. (2013). Enseñanzaaprendizaje desarrollador escuela primaria, Teoría y práctica. La Habana: Pueblo y Educación
Rojas, D. (2011). Percepción de Alimentación Saludable, Hábitos Alimentarios Estado Nutricional y Práctica de Actividad Física en Población de 9-11 años del Colegio Cedid Ciudad Bolívar, Bogotá. http://www.javeriana.edu.co/ biblos/tesis/ciencias/tesis704.pdf

Torres-Zapata, A. E., Solis-Cardouwer, O. C., Rodríguez-Rosas, C., Moguel-Ceballos, J. E., y Zapata-Gerónimo, D. (2017). Hábitos alimentarios y estado nutricional en trabajadores de la industria petrolera. Horizonte sanitario, 16(3), 183-190. http://dx.doi. org/10.19136/hs.a16n3.1788

Vygotsky, L. (1978). Zona de desarrollo próximo: una nueva aproximación. El desarrollo de los procesos psicológicos superiores. Barcelona: Grijalbo

Zegarra, R. y Velázquez-Tejeda, M. E. (2016). El coaching: una forma para fortalecer el profesionalismo del docente en el aula. Páginas de educación, 9(2). doi: https://doi.org/10.22235/ pe.v9i2.1294 